\title{
Adaptive Fuzzy Sliding Mode Power System Stabilizer Using Nussbaum Gain
}

\author{
Emira Nechadi ${ }^{1} \quad$ Mohamed Naguib Harmas $^{1} \quad$ Najib Essounbouli $^{2} \quad$ Abdelaziz Hamzaoui $^{2}$ \\ ${ }^{1}$ Electrical Engineering Department, Ferhat Abbas University of Sétif 1, Algeria \\ ${ }^{2}$ Centre of Research for Science and Information Technology and Communication Laboratory, Champagne Ardennes University, France
}

\begin{abstract}
Power system stability is enhanced through a novel stabilizer developed around an adaptive fuzzy sliding mode approach which applies the Nussbaum gain to a nonlinear model of a single-machine infinite-bus (SMIB) and multi-machine power system stabilizer subjected to a three phase fault. The Nussbaum gain is used to avoid the positive sign constraint and the problem of controllability of the system. A comparative simulation study is presented to evaluate the achieved performance.
\end{abstract}

Keywords: Multi-machine power system stabilizer, adaptive fuzzy, sliding mode, Nussbaum gain, Lyapounov stability.

\section{Introduction}

Power systems are complex nonlinear systems that often exhibit low frequency oscillations due to insufficient damping caused by adverse operating conditions which can lead to a devastating loss of synchronism ${ }^{[1]}$. Power system stabilizers are used to suppress these oscillations and improve the overall stability ${ }^{[1-4]}$. The computation of the fixed parameters of these stabilizers is usually based on the linearized model of the power system around a nominal operating point ${ }^{[5-7]}$. Operating conditions often change as a result of load variations and/or major disturbances. These changes affect power system dynamic behavior which requires adjustment of stabilizer parameters. Keeping the latter at fixed values will greatly degrade power system performance $^{[7]}$. Conventional stabilizers, using lead-lag compensators have been based on linearized power system model to damp oscillations. Disturbances, varying loading conditions and therefore frequently changing operating point were not taken into consideration ${ }^{[8-11]}$. However, a lot of researches about the design of power system stabilizers have been conducted, using a wide range of strategies, such as sliding controller ${ }^{[12]}$, adaptive controller ${ }^{[13-15]}$, and adaptive fuzzy controllers ${ }^{[16,17]}$. A comparison of some approaches to designing power system stabilizers was presented in [18]. One of these possible methods is the application of adaptive fuzzy sliding controller. Remarkable research effort has been done in the last decade to put forward intelligent fuzzy logic based power system stabilizer (PSS) as well as optimality in adapting to changing operating conditions as in [19-21]. However, these linear model based control strategies often fail to provide satisfactory results over a wide range of operating conditions. Moreover, during severe disturbances, PSS action may actually cause the generator under its control to lose synchronism in an attempt to control its excitation field.

For the last few years, optimization techniques for a conventional ${ }^{[22-23]}$ and dual $\operatorname{PSS}^{[24-26]}$ have also been applied using different algorithms such as particle swarm optimization (PSO), genetic algorithms (GA), chaotic opti- mization algorithm (COA) and neuro-fuzzy system (NFS).

Contribution made in this paper consists in a new adaptive fuzzy sliding mode power system stabilizer using a Nussbaum gain. Stability of the overall system is guaranteed via Lyapunov synthesis.

In the following sections of this paper, a nonlinear power system model is presented first, followed by the development of an adaptive fuzzy sliding mode controller using Nussbaum gain and the addressed stability issue. In Section 3 , the study of simulation results for different operating conditions on single-machine and multi-machine power system is described.

\section{Power system model}

The power system model considered in this paper is a nonlinear model representing a synchronous machine connected to an infinite bus via a double circuit transmission line. Fig. 1 shows the power system schematic diagram including turbine, transformer, automatic voltage regulator and $\operatorname{PSS}^{[4]}$.

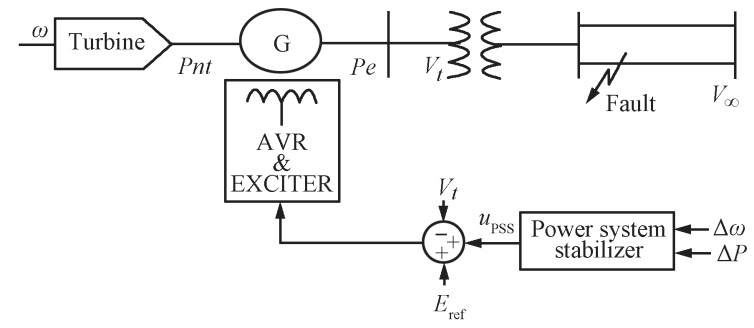

Fig. 1 Single-machine infinite-bus (SMIB) power system

A nonlinear representation of the power system during a transient period after a major disturbance has occurred in the system ${ }^{[17,27,28]}$ :

$$
\left\{\begin{array}{l}
\dot{x}_{1}=x_{2} \\
\dot{x}_{2}=f(x)+g(x) u
\end{array}\right.
$$

where $x=\left[\begin{array}{ll}x_{1} & x_{2}\end{array}\right]^{\mathrm{T}}=\left[\begin{array}{cc}\Delta \omega & \frac{\Delta P}{M}\end{array}\right]^{\mathrm{T}} \in \mathbf{R}^{2}$ is the 
state vector, $\Delta \omega$ is the speed deviation, $\Delta P=P_{m}-P_{e}$ is the accelerating power, $M$ is inertia moment coefficient of the synchronous machine, $u \in \mathbf{R}$ is the input, $f(x)$ and $g(x)$ are nonlinear functions, and $g(x) \neq 0$ in the controllability region (see the appendix).

The block diagram of a conventional lead-lag power system stabilizer is shown in Fig. 2, in which a conventional single-input is presented. Parameters and details can be found in [22].

The two inputs of an IEEE power system stabilizer, unlike the conventional single-input PSS, is shown in Fig. 3, in which a dual-input PSS3B is presented. Parameters and details can be found in [24].

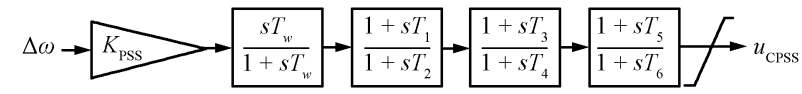

Fig. 2 Conventional power system stabilizer

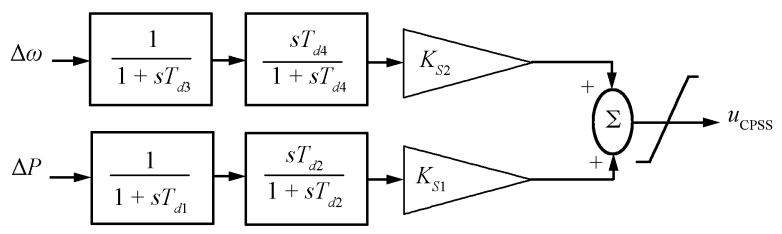

Fig. 3 Dual-input power system stabilizer

\section{Adaptive fuzzy sliding mode power system stabilizer}

Consider a single-input-single-output (SISO) nonlinear system described by (1), with the sliding surface as

$$
S=x_{2}+\beta x_{1}
$$

where $\beta$ is a positive constant.

The time derivative of the sliding surface (2) is given by

$$
\dot{S}=f(x)+\beta x_{2}+g(x) u .
$$

Assuring the existing condition (4)

$$
S \dot{S}<0
$$

Hence, the control law is

$$
u=g^{-1}(x)\left(-f(x)-\beta x_{2}-k \operatorname{sgn}(S)\right) .
$$

Control law (5) allows ensuring the system stabilization and robustness, but it has the drawback in the computation of $k$, which is not a straightforward task. In a more realistic case where $f(x)$ and $g(x)$ are unknown, they are replaced by their fuzzy estimations.

In previous researches on the indirect adaptive fuzzy method, the controller with $\hat{g}^{-1}(x)$ can be singular because it cannot be guaranteed that $\hat{g}(x)$ is not equal to zero at any moment, where $\hat{g}(x)$ denotes the approximation of $g(x)$. In this paper, a Nussbaum gain will be incorporated into the control law in order to estimate function $\hat{g}^{-1}(x)^{[29]}$.
Definition $\mathbf{1}^{[30]}$. A function is called a Nussbaum-type function if it has the following properties:

$$
\begin{aligned}
& \lim _{y \rightarrow \infty} \sup \frac{1}{y} \int_{0}^{y} N(\varsigma) \mathrm{d} \varsigma=+\infty \\
& \lim _{y \rightarrow \infty} \inf \frac{1}{y} \int_{0}^{y} N(\varsigma) \mathrm{d} \varsigma=-\infty .
\end{aligned}
$$

Throughout this paper, the even Nussbaum function:

$$
N(\varsigma)=\cos \left(\frac{\pi}{2}\right) \varsigma \mathrm{e}^{\varsigma^{2}}
$$

is employed, and $\varsigma$ is a variable to be determined later.

Lemma $1^{[30]}$. Let $V(\cdot)$ and $\varsigma(\cdot)$ be smooth functions defined on $\left[0, t_{f}\right)$, with $V(t) \geqslant 0, \forall t \in\left[0, t_{f}\right)$, and $N(\cdot)$ be an even Nussbaum-type function. If the following inequality holds:

$$
\begin{aligned}
V(t) \leqslant & c_{0}+\mathrm{e}^{-c_{1} t} \int_{0}^{t} g^{\prime}(x(\tau)) N(\varsigma) \dot{\varsigma} \mathrm{e}^{c_{1} \tau} \mathrm{d} \tau+ \\
& \mathrm{e}^{-c_{1} t} \int_{0}^{t} \dot{\varsigma} \mathrm{e}^{c_{1} \tau} \mathrm{d} \tau, \forall t \in\left[0, t_{f}\right)
\end{aligned}
$$

where $c_{0}$ represents some suitable constant, $c_{1}$ is a positive constant, and $g^{\prime}(x(\tau))$ is a time-varying parameter which takes values in the unknown closed interval $I=\left[l^{-}, l^{+}\right]$, with $0 \notin I$, then $V(t), \varsigma(t)$ and $\int_{0}^{t} g^{\prime}(x(\tau)) N(\varsigma) \dot{\varsigma} \mathrm{d} \tau$ must be bounded on $\left[0, t_{f}\right)$.

The proof of this lemma can be found in [30].

Based on the universal approximation theorem, the unknown function $f(x)$ and constant $k$ can be approximated by (10) and (11), respectively:

$$
\begin{aligned}
& \hat{f}\left(x, \theta_{f}\right)=\xi^{\mathrm{T}}(x) \theta_{f} \\
& \hat{k}\left(x, \theta_{k}\right)=\xi^{\mathrm{T}}(x) \theta_{k}
\end{aligned}
$$

where $\theta=\left[\theta_{1}, \theta_{2}, \cdots, \theta_{m}\right]^{\mathrm{T}}$ is the parameter vector and $\xi=\left[\xi_{1}, \xi_{2}, \cdots, \xi_{m}\right]^{\mathrm{T}}$ is the vector of fuzzy basis functions.

The approximation error is given by

$$
\begin{gathered}
\delta_{f}=f(x)-\xi^{\mathrm{T}}(x) \theta_{f}^{*} \\
\delta_{k}=k-\xi^{\mathrm{T}}(x) \theta_{k}^{*}
\end{gathered}
$$

in which $\theta_{f}^{*}$ and $\theta_{k}^{*}$ are the optimal parameter values. We define

$$
\begin{aligned}
& \tilde{\theta}_{f}=\theta_{f}-\theta_{f}^{*} \\
& \tilde{\theta}_{k}=\theta_{k}-\theta_{k}^{*} .
\end{aligned}
$$

Theorem 1. For the nonlinear system (1), if we choose the following control law

$$
u=N(\varsigma)\left(-\hat{f}(x)-\beta x_{2}-\hat{k} \operatorname{sgn}(S)\right)
$$

with

$$
\dot{\varsigma}=S\left(\theta_{f}^{\mathrm{T}} \xi(x)+\beta x_{2}\right)
$$

and the following adaptation laws:

$$
\begin{gathered}
\dot{\theta}_{f}=\gamma_{1} S \xi(x)-\gamma_{1} \theta_{f} \\
\dot{\theta}_{k}=\gamma_{2} g(x) N(\varsigma)|S| \xi(x)-\gamma_{2} \theta_{k}
\end{gathered}
$$


such that $\left|\delta_{f} S\right| \leqslant \varepsilon, \gamma_{1}, \gamma_{2}$ are positive constants and $g^{\prime}(x)=-g(x)$, then the stability of the closed loop system can be guaranteed.

Proof. Choose the Lyapunov function candidate to be

$$
V=\frac{1}{2} S^{\mathrm{T}} S+\frac{1}{2 \gamma_{1}} \tilde{\theta}_{f}^{\mathrm{T}} \tilde{\theta}_{f}+\frac{1}{2 \gamma_{2}} \tilde{\theta}_{k}^{\mathrm{T}} \tilde{\theta}_{k} .
$$

Therefore,

$$
\begin{aligned}
\dot{V} & =S^{\mathrm{T}} \dot{S}+\frac{1}{\gamma_{1}} \tilde{\theta}_{f}^{\mathrm{T}} \dot{\theta}_{f}+\frac{1}{\gamma_{2}} \tilde{\theta}_{k}^{\mathrm{T}} \dot{\theta}_{k}= \\
& S^{\mathrm{T}}\left(f(x)+g(x) u+\beta x_{2}\right)+\frac{1}{\gamma_{1}} \tilde{\theta}_{f}^{\mathrm{T}} \dot{\theta}_{f}+\frac{1}{\gamma_{2}} \tilde{\theta}_{k}^{\mathrm{T}} \dot{\theta}_{k}= \\
& S^{\mathrm{T}}\left(\left(g^{\prime}(x) N(\varsigma)+1\right)\left(\theta_{f}^{\mathrm{T}} \xi(x)+\beta x_{2}\right)-\tilde{\theta}_{f}^{\mathrm{T}} \xi(x)+\delta_{f}\right)- \\
& \hat{k} g(x) N(\varsigma) \operatorname{sgn}(S))+\frac{1}{\gamma_{1}} \tilde{\theta}_{f}^{\mathrm{T}} \dot{\theta}_{f}+\frac{1}{\gamma_{2}} \tilde{\theta}_{k}^{\mathrm{T}} \dot{\theta}_{k}= \\
& {\left[g^{\prime}(x) N(\varsigma)+1\right] \dot{\varsigma}-\tilde{\theta}_{f}^{\mathrm{T}} S \xi(x)+\delta f S-\frac{k^{*} g(x) N(\varsigma)}{|S|} S^{\mathrm{T}} S-} \\
& \tilde{\theta}_{k}^{\mathrm{T}} g(x) N(\varsigma)|S| \xi(x)+\frac{1}{\gamma_{1}} \tilde{\theta}_{f}^{\mathrm{T}} \dot{\theta}_{f}+\frac{1}{\gamma_{2}} \tilde{\theta}_{k}^{\mathrm{T}} \dot{\theta}_{k} .
\end{aligned}
$$

Using (18) and (19), we can obtain

$$
\begin{aligned}
\dot{V}=- & \frac{k^{*} g(x) N(\varsigma)}{|S|} S^{\mathrm{T}} S-\theta_{f}^{\mathrm{T}} \tilde{\theta}_{f}- \\
& \theta_{k}^{\mathrm{T}} \tilde{\theta}_{k}+\delta_{f} S+\left(g^{\prime}(x) N(\varsigma)+1\right) \dot{\varsigma} .
\end{aligned}
$$

The following inequalities are valid

$$
\begin{aligned}
& -\theta_{f}^{\mathrm{T}} \tilde{\theta}_{f} \leqslant-\frac{1}{2} \tilde{\theta}_{f}^{\mathrm{T}} \tilde{\theta}_{f}+\frac{1}{2}\left\|\theta_{f}^{*}\right\|^{2} \\
& -\theta_{k}^{\mathrm{T}} \tilde{\theta}_{k} \leqslant-\frac{1}{2} \tilde{\theta}_{k}^{\mathrm{T}} \tilde{\theta}_{k}+\frac{1}{2}\left\|\theta_{k}^{*}\right\|^{2} .
\end{aligned}
$$

And we can rewrite $\dot{V}$ as

$$
\begin{gathered}
\dot{V} \leqslant-\frac{k^{*} g(x) N(\varsigma)}{|S|} S^{\mathrm{T}} S-\frac{1}{2} \tilde{\theta}_{f}^{\mathrm{T}} \tilde{\theta}_{f}-\frac{1}{2} \tilde{\theta}_{k}^{\mathrm{T}} \tilde{\theta}_{k}+\frac{1}{2}\left\|\theta_{f}^{*}\right\|^{2}+ \\
\frac{1}{2}\left\|\theta_{k}^{*}\right\|^{2}+\|\varepsilon\|+\left(g^{\prime}(x) N(\varsigma)+1\right) \dot{\varsigma}
\end{gathered}
$$

Let $\alpha=\min \left\{\lambda_{\min }\left(\frac{2 k^{*} g(x) N(\varsigma)}{|s|}\right), \lambda_{\min }\left(\gamma_{1}\right), \lambda_{\min }\left(\gamma_{2}\right)\right\}$ and $\beta=\frac{1}{2}\left\|\theta_{f}^{*}\right\|^{2}+\frac{1}{2}\left\|\theta_{k}^{*}\right\|^{2}+\|\varepsilon\|$. Then, (25) becomes

$$
\dot{V} \leqslant-\alpha V+\beta+\left(g^{\prime}(x) N(\varsigma)+1\right) \dot{\varsigma}
$$

Multiplying both sides of (26) by $\mathrm{e}^{\alpha t}$, we can obtain

$$
\frac{\mathrm{d}}{\mathrm{d} t}\left(V(t) \mathrm{e}^{\alpha t}\right) \leqslant \beta \mathrm{e}^{\alpha t}+\mathrm{e}^{\alpha t}\left[g^{\prime}(x) N(\varsigma)+1\right] \dot{\varsigma} .
$$

After integrating $(27)$ over $\left[0, t_{f}\right]$, it follows that

$$
\begin{aligned}
& 0 \leqslant V(t) \leqslant \frac{\beta}{\alpha}+\left[V(0)-\frac{\beta}{\alpha}\right] \mathrm{e}^{-\alpha t}+ \\
& \mathrm{e}^{-\alpha t} \int_{0}^{t}\left[g^{\prime}(x) N(\varsigma)+1\right] \mathrm{e}^{\alpha \tau} \dot{\varsigma} \mathrm{d} \tau .
\end{aligned}
$$

Noting that $0<\mathrm{e}^{-\alpha t}<1$ and $\frac{\beta}{\alpha} \mathrm{e}^{-\alpha t}>0$, we have $\left[V(0)-\frac{\beta}{\alpha}\right] \mathrm{e}^{-\alpha t} \leqslant V(0)$. Then the above equality becomes

$$
0 \leqslant V(t) \leqslant \eta+\mathrm{e}^{-\alpha t} \int_{0}^{t}\left[g^{\prime}(x) N(\varsigma)+1\right] \mathrm{e}^{\alpha \tau} \dot{\varsigma} \mathrm{d} \tau
$$

where $\eta=\frac{\beta}{\alpha}+V(0)$.

According to Lemma 1, it can be concluded from (32) that $V(t)$ and $\int_{0}^{t} g^{\prime}(x) N(\varsigma) \dot{\varsigma} \mathrm{d} \tau$ are bounded, i.e., $S, \tilde{\theta}_{f}, \tilde{\theta}_{k}, \varsigma(t)$ and $\int_{0}^{t}\left[g^{\prime}(x) N(\varsigma)+1\right] \mathrm{e}^{\alpha \tau} \dot{\varsigma} \mathrm{d} \tau$ are also bounded.
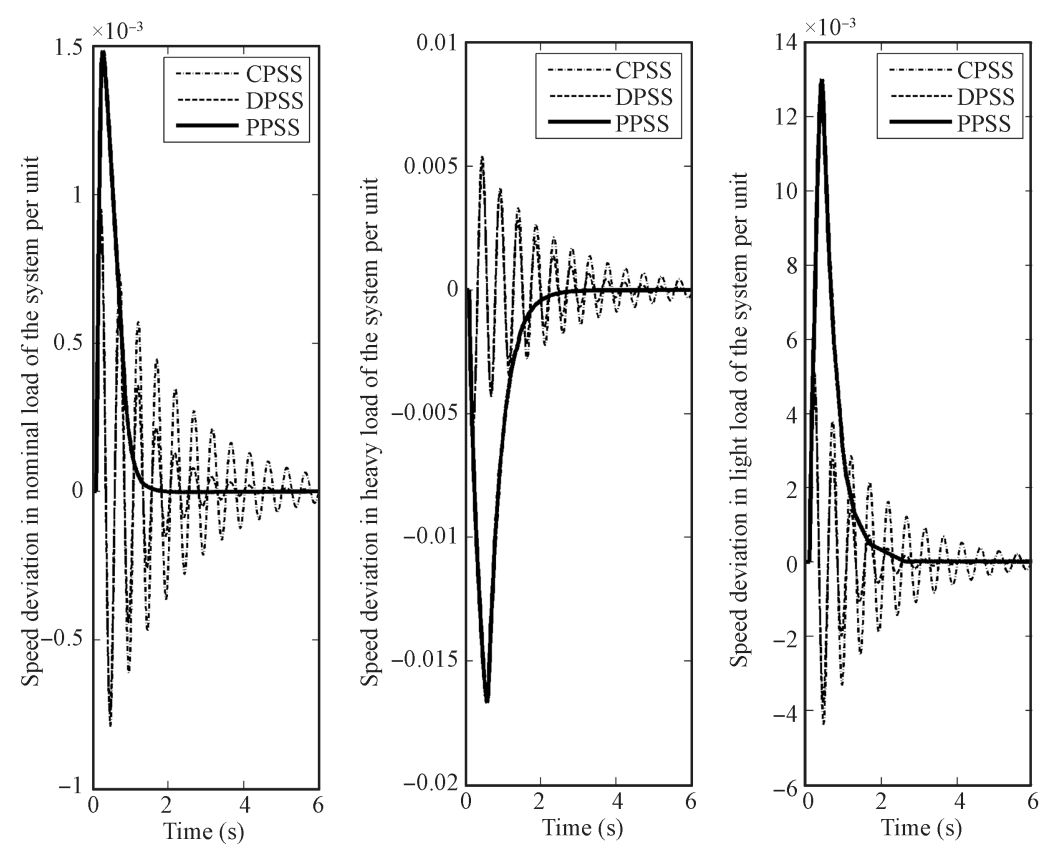

Fig. 4 Speed deviations in nominal, heavy loading and light loading cases 


\section{Simulation}

The soundness of the proposed PSS was tested, and the performance as well as robustness tests were conducted and compared with a conventional stabilizer and a dual-input power system stabilizer through simulations. Good transient behavior with the proposed control under severe operating conditions were illustrated by the following case studies. The speed variation and accelerating power are chosen as the power system control variables. Five fuzzy sets for each input are sufficient for the PSS to be designed.

\subsection{Simulation cases for an SMIB}

To assess the performance of the proposed controller, simulations were carried out for different operating conditions.

A three-phase fault test is applied to an infinite bus as in Fig. 1, lasting $60 \mathrm{~ms}$ before being cleared. When the power system is strongly perturbed, the proposed stabilizer reacts rapidly and prevents an eventual loss of synchronism. Therefore, it enables the system to reach a stable operating point very quickly.

The simulation results for nominal load, heavy load and light load are shown in Fig. 4. It is clear that the proposed PSS (PPSS) exhibits superior performance to the conventional PSS (CPSS) and dual-input PSS (DPSS) power system stabilizers. The simulation results shown in Fig. 4 indicates a good transient behavior of the proposed PSS.

\subsection{Simulation of multi-machine power system}

To evaluate the performance of the proposed control, we performed simulation for multi-machine power system as in Fig. 5 with the aim to compare the performance of the proposed PSS with the conventional PSS and dual-input PSSs.

In order to validate stability enhancement due to the proposed stabilizer, a three-phase fault test is applied to bus 7 (Fig. 5) with a duration of $60 \mathrm{~ms}$ before it is cleared. Three operating conditions were investigated: Cases of nominal load, light load and heavy load. The fault is cleared and the controller helps the system to reach a stable operating point very quickly. As shown in Fig. 6, the proposed approach shows better control performance than the conventional PSS and dual PSSs in terms of settling time and damping effect.

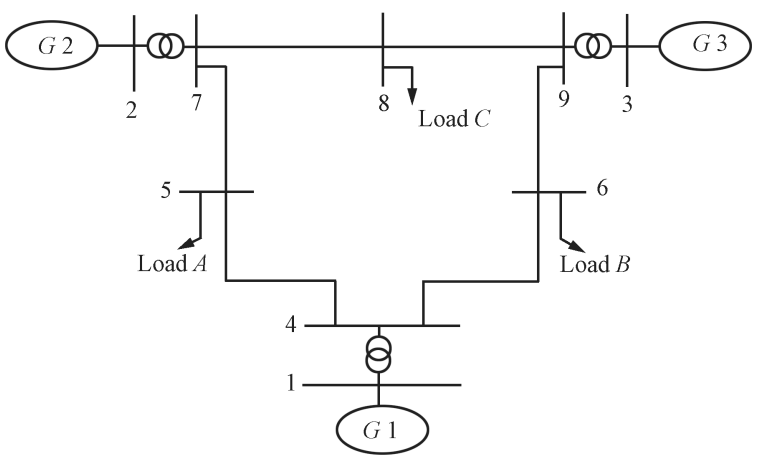

Fig. 5 Multi-machine power system

To further evaluate the robustness of the proposed stabilizer, the power system is subjected to heavy load operation (Fig. 7) and light load operation (Fig. 8). The results show again the clear oscillations damping of the proposed controller in multi-machine power system, as compared with its conventional counterpart, but we have a small deterioration in performance.
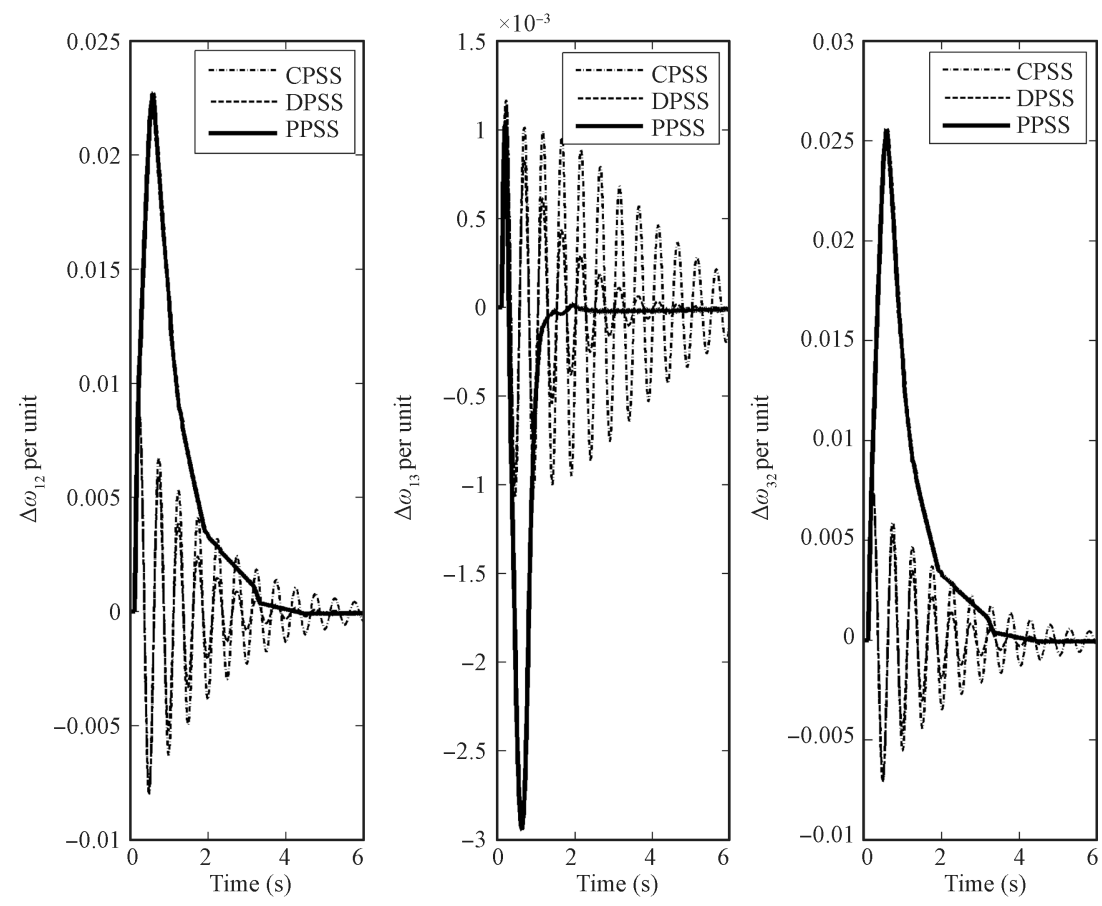

Fig. 6 Speed deviations in nominal case 

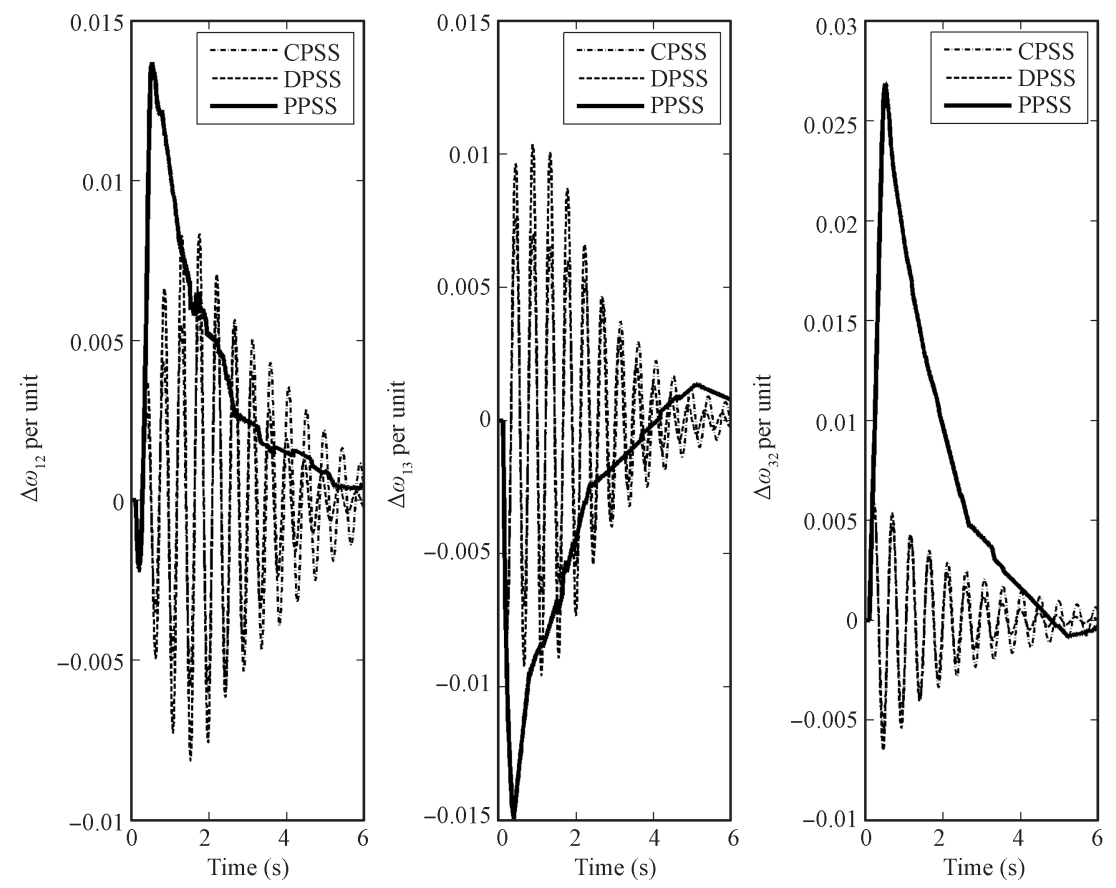

Fig. 7 Speed deviations in heavy loading of the system
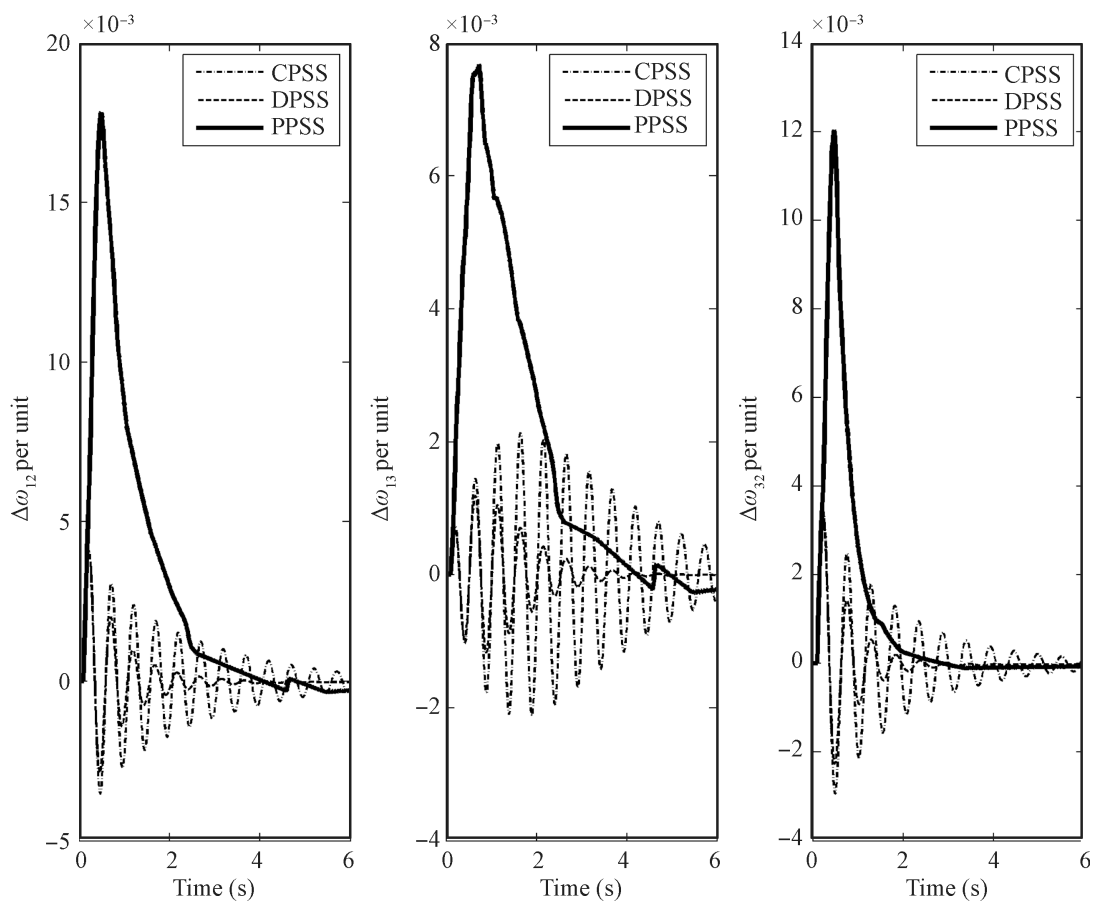

Fig. 8 Speed deviations in light loading of the system

In this study, we used different operating points to demonstrate the effectiveness of the proposed control in oscillations damping after the occurrence of large disturbance on a power system by providing better transient response and stronger robustness than other stabilizers. The adaptive fuzzy sliding mode using Nussbaum gain scheme permits to take account of severe load variation and varying operating conditions.

\section{Conclusions}

Based on the adaptive fuzzy sliding mode controller and the Nussbaum gain, we introduced a new power system stabilizer that enhances damping and improves transient dynamics of a single-machine infinite-bus and multi-machine power system stabilizers. Different load conditions as well as severe perturbations were used to evaluate the proposed 
power system stabilizer effectiveness in rapidly reducing oscillations that could lead to loss of synchronism if not treated. Simulation results exhibited its superior performance over classical PSSs. Real power system remains to be thoroughly investigated under the proposed stabilizer.

\section{Appendix}

Parameters of single (SMIB) operating conditions:

Nominal load: $P=0.9 /$ unit, $Q=0.3 /$ unit

Heavy load: $P=1.60 /$ unit, $Q=0.6 /$ unit

Light load: $P=0.45 /$ unit, $Q=0.35 /$ unit

Multi-machine power system parameters:

G1:Nominal load: $P=0.72 /$ unit, $Q=0.27 /$ unit.

Heavy load: $P=2.21 /$ unit, $Q=1.09 /$ unit.

Light load: $P=0.36 /$ unit, $Q=0.16 /$ unit.

G2:Nominal load: $P=1.63 /$ unit, $Q=0.07 /$ unit.

Heavy load: $P=1.92 /$ unit, $Q=0.56 /$ unit.

Light load: $P=0.80 /$ unit, $Q=-0.11 /$ unit.

G3:Nominal load: $P=0.85 /$ unit, $Q=-0.11 /$ unit.

Heavy load: $P=1.28 /$ unit, $Q=0.36 /$ unit.

Lightload: $P=0.45 /$ unit, $Q=-0.20 /$ unit.

Nonlinear functions:

$$
\begin{aligned}
& f(x)=\frac{x_{e}+x_{d}}{T_{d 0}^{\prime}\left(x_{e}+x_{d}^{\prime}\right)}\left(\Delta P-P_{m}\right)+\frac{V^{2}}{2 T_{d 0}^{\prime}\left(x_{e}+x_{d}^{\prime}\right)^{2}} \\
& \left(\frac{\left(x_{e}+x_{d}\right)\left(x_{d}^{\prime}-x_{q}\right)}{\left(x_{e}+x_{q}\right)}+\left(x_{d}-x_{d}^{\prime}\right)\right) \sin (2 \delta)+ \\
& \left(Q+V^{2}\left(\frac{\sin ^{2}(\delta)}{x_{e}+x_{q}}+\frac{\cos ^{2}(\delta)}{x_{e}+x_{d}^{\prime}}+\frac{\left(x_{d}^{\prime}-x_{q}\right) \cos (2 \delta)}{\left(x_{e}+x_{q}\right)\left(x_{e}+x_{d}^{\prime}\right)}\right)\right) \times \\
& \omega_{s m} \Delta \omega+\frac{K_{A} V \sin (\delta)}{T_{d 0}^{\prime}\left(x_{e}+x_{d}^{\prime}\right)}\left(V_{r e f}-V_{t}\right) \\
& g(x)=\frac{K_{A} V \sin (\delta)}{T_{d 0}^{\prime}\left(x_{e}+x_{d}^{\prime}\right)} .
\end{aligned}
$$

\section{References}

[1] P. Kundur. Power System Stability and Control, New York, USA: McGraw-Hill Inc, 1994.

[2] F. P. DeMello, C. A. Concordia. Concepts of synchronous machine stability as affected by excitation control. IEEE Transactions on Power Apparatus and Systems, vol. PAS88, no. 4, pp. 316-329, 1969.

[3] P. M. Anderson, A. A. Fouad. Power System Control and Stability, Lowa State, USA: IEEE Press, 1977.

[4] E. V. Larsen, D. A. Swann. Applying power system stabilizers, Part I, II, III. IEEE Transactions on Power Apparatus and Systems, vol. PAS-100, no. 6, pp. 3017-3046, 1981.

[5] M. L. Kothari, J. Nanda, K. Bhattacharya. Design of variable structure power system stabilisers with desired eigenvalues in the sliding mode. IEE Proceedings $C$ of Generation, Transmission and Distribution, vol. 140, no. 4, pp. 263-268, 1993.

[6] K. Bhattacharya, M. L Kothari, J. Nanda. Design of discrete-mode variable structure power system stabilizers. International Journal of Electrical Power \& Energy Systems, vol. 17, no. 6, pp. 399-406, 1995.
[7] Y. M. Park, W. Kim. Discrete-time adaptive sliding mode power system stabilizer with only input/output measurements. International Journal of Electrical Power \& Energy Systems, vol.18, no. 8, pp. 509-517, 1996.

[8] Z. H. Jiang. Design of power system stabilizers using synergetic control theory. In Proceedings of Power Engineering Society General Meeting, IEEE, Tampa, FL, pp. 1-8, 2007.

[9] A. L. Elshafei, K. A. El-Metwally, A. A. Shaltout. A variable structure adaptive fuzzy logic stabilizer for single and multi-machine power systems. Control Engineering Practice, vol. 13, no. 4, pp. 413-423, 2005.

[10] P. Hoang, K. Tomsovic. Design and analysis of an adaptive fuzzy power system stabilizer. IEEE Transactions on Energy Conversion, vol. 11, no. 2, pp. 455-461, 1996.

[11] G. J. Li, T. T. Lie, C. B. Soh, G. H. Yang. Design of statefeedback decentralized nonlinear $H_{\infty}$ controllers in power systems. International Journal of Electrical Power \& Energy Systems, vol. 24, no. 8, pp.601-610, 2002.

[12] A. Y. Sivaramakrishnan, M. V. Hariharan, M. C. Srisailam. Design of variable-structure load-frequency controller using pole assignment technique. International Journal of Control, vol. 40, no. 3, pp. 487-498, 1984.

[13] A. Ghosh, G. Ledwich, O. P. Malik, G. S. Hope. Power system stabilizer based on adaptive control techniques. IEEE Transactions on Power Apparatus and Systems, vol. PAS103, no. 8, pp. 1983-1989, 1984.

[14] S. J. Cheng, Y. S. Chow, O. P. Malik, G. S. Hope. An adaptive synchronous machine stabilizer. IEEE Transactions on Power Systems, vol. 1, no. 3, pp.101-107, 1986.

[15] D. A. Pierre. A perspective on adaptive control of power systems. IEEE Transactions on Power Systems, vol. 2, no. 2, pp. 387-395, 1987.

[16] N. Hossein-Zadeh, A. Kalam. A direct adaptive fuzzy power system stabilizer. IEEE Transactions on Energy Conversion, vol. 14, no. 4, pp. 1564-1571, 1999.

[17] N. Hossein-Zadeh, A. Kalam. An indirect adaptive fuzzylogic power system stabiliser. International Journal of Electrical Power \& Energy Systems, vol. 24, no. 10, pp. 837-842, 2002 .

[18] A. L. Elshafei, K. El-Metwally. Power system stabilization via adaptive fuzzy-logic control. In Proceedings of the 12th IEEE International Symposium on Intelligent Control, IEEE, Istanbul, Turkey, pp. 89-94, 1997.

[19] S. S. Lee, J. K. Park. Design of power system stabilizer using observer/sliding mode, observer/sliding mode-model following and $H_{\infty} /$ sliding mode controllers for small-signal stability study. International Journal of Electrical Power \& Energy Systems, vol. 20, no. 8, pp. 543-553, 1998.

[20] T. Hussein, M. S. Saad, A. L. Elshafei, A. Bahgat. Damping inter-area modes of oscillation using an adaptive fuzzy power system stabilizer. Electric Power Systems Research, vol. 80, no. 12, pp. 1428-1436, 2010.

[21] G. H. Hwang, D. W. Kim, J. H. Lee, Y. J. An. Design of fuzzy power system stabilizer using adaptive evolutionary algorithm. Engineering Applications of Artificial Intelligence, vol. 21, no. 1, pp. 86-96, 2008. 
[22] M. Soliman, A. L. Elshafei, F. Bendary, W. Mansour. LMI static output-feedback design of fuzzy power system stabilizers. Expert Systems with Applications, vol.36, no. 3, pp. 6817-6825, 2009.

[23] H. Shayeghi, H. A. Shayanfar, A. Safari, R. Aghmasheh. A robust PSSs design using PSO in a multi-machine environment. Energy Conversion and Management, vol. 51, no. 4, pp. 696-702, 2010.

[24] H. Shayeghi, H. A. Shayanfar, S. Jalilzadeh, A. Safari. Multi-machine power system stabilizers design using chaotic optimization algorithm. Energy Conversion and Management, vol. 51, no. 7, pp. 1572-1580, 2010.

[25] A. Chatterjee, S. P. Ghoshal, V. Mukherjee. Chaotic ant swarm optimization for fuzzy-based tuning of power system stabilizer. International Journal of Electrical Power and Energy Systems, vol 33, no. 3, pp. 657-672, 2011.

[26] A. Sharma, M. L. Kothari. Intelligent dual input power system stabilizer. Electric Power Systems Research, vol. 64, no. 3, pp. 257-267, 2003.

[27] S. P. Ghoshal, A. Chatterjee, V. Mukherjee. Bio-inspired fuzzy logic based tuning of power system stabilizer. Expert Systems with Applications, vol.36, no. 5, pp.9281-9292, 2009.

[28] M. A. M. Hassan, O. P. Malik, G. S. Hope. A fuzzy logic based stabilizer for a synchronous machine. IEEE Transactions on Energy Conversion, vol. 6, no. 3, pp. 407-413, 1991.

[29] A. Boulkroune, M. Tadjine, M. MSaad, M. Farza. Fuzzy adaptive controller for MIMO nonlinear systems with known and unknown control direction. Fuzzy Sets and Systems, vol. 161, no. 6, pp. 797-820, 2010.

[30] S. S. Ge, H. Fan, T. H. Lee. Adaptive neural control of nonlinear time-delay systems with unknown virtual control coefficients. IEEE Transactions on Systems, Man, and Cybernetics-Part B: Cybernetics, vol. 34, no. 1, pp. 499516, 2004.

Emira Nechadi received her bachelor degree in control systems, master degree in control systems, and Ph. D. degree in control systems, all from the University of Sétif, Algeria. in 2002 , 2004, and 2013, respectively.

Her research interests include sliding mode control, adaptive fuzzy control, fuzzy systems, synergetic control and power systems.

E-mail: nechadiamira@yahoo.fr (Corresponding author)

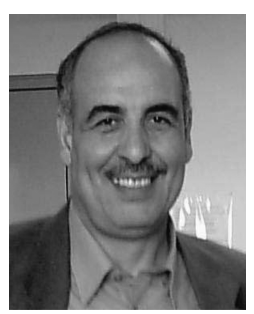

Mohamed Naguib Harmas received the $\mathrm{Ph} . \mathrm{D}$. degree in control systems from Ferhat Abbas University of Sétif 1, and M. Sc. degree in electrical engineering and electronics from California State University of Sacramento, Sacramento, USA. He is currently a professor at Sétif University, Algeria.

His research interests include robust nonlinear control, power system and power electronics control, and fuzzy synergetic design.

E-mail: mharmas@yahoo.fr

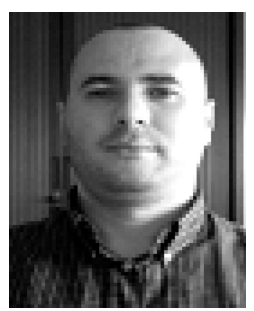

Najib Essounbouli received his bachelor degree in electrical from the University of Sciences and Technology of Marrakech (FSTG) Morocco, and DEA. and Ph.D. degrees both in electrical engineering from Reims University of Champagne Ardennes, in 2000 and 2004 respectively. From 2005 to 2010, he was an assistant professor with University Institute of Troyes, Reims Champagne Ardennes University. Since 2010, he has been a professor with the same institute.

His research interests include fuzzy logic control, robust adaptive control, renewable energy and control drive.

E-mail: najib.essounbouli@univ-reims.fr

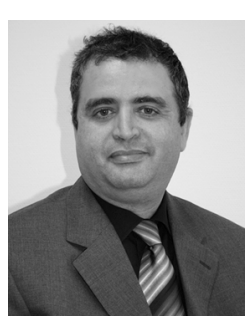

Abdelaziz Hamzaoui received his bachelor degree in electrical engineering from the Polytechnic School of Algiers (ENPA), Algeria, in 1982, and DEA. and $\mathrm{Ph}$. D. degrees both in electrical engineering from Reims University of Champagne Ardennes, in 1989 and 1992, respectively. He is currently a professor and the director of the Technology Institute of Troyes, Reims Champagne Ardennes University.

His research interests include intelligent control, fuzzy control, and robust adaptive control.

E-mail: abdelaziz.hamzaoui@univ-reims.fr 